\title{
Electron Screening and Kinetic-Energy Oscillations in a Strongly Coupled Plasma
}

\author{
Y. C. Chen, C. E. Simien, S. Laha, P. Gupta, Y. N. Martinez, P. G. Mickelson, S. B. Nagel, and T. C. Killian \\ Department of Physics and Astronomy, Rice University, Houston, Texas 77005, USA \\ and Rice Quantum Institute, Rice University, Houston, Texas 77005, USA
}

(Received 24 August 2004; revised manuscript received 10 December 2004; published 20 December 2004)

\begin{abstract}
We study equilibration of strongly coupled ions in an ultracold neutral plasma produced by photoionizing laser-cooled and trapped atoms. By varying the electron temperature, we show that electron screening modifies the equilibrium ion temperature. Even with few electrons in a Debye sphere, the screening is well described by a model using a Yukawa ion-ion potential. We also observe damped oscillations of the ion kinetic energy that are a unique feature of equilibration of a strongly coupled plasma.
\end{abstract}

\section{DOI: 10.1103/PhysRevLett.93.265003}

A plasma becomes strongly coupled when the electrical interaction energy between the charged particles exceeds the thermal energy. Strong coupling is observed in dense astrophysical environments [1], such as the interiors of gas giant planets, and in laser-cooled ions [2], dusty plasmas [3], and solid targets [4] and clusters [5] irradiated by pulsed high-intensity lasers. Equilibration in these systems is of particular interest because it involves the establishment of spatial correlations between particles.

There has been significant theoretical study of the equilibration of strongly coupled plasmas [6-12], especially in the context of plasmas produced with high-intensity lasers. In addition to generating fundamental interest, this problem challenges computational resources and techniques. Experimental results have been lacking, however, because of the fast time scales involved and limited diagnostics.

Ultracold neutral plasmas [13], produced by photoionizing clouds of laser-cooled and trapped atoms, are ideal for experimental studies. The equilibration of the plasma is relatively slow $(\sim 100 \mathrm{~ns})$ due to lower plasma density. Ultracold neutral plasmas also offer a high level of control and diagnostics. By varying laser intensities and wavelengths, it is possible to accurately set the initial density and energy of the system. Optical imaging [14] provides an in situ probe of plasma properties with excellent spatial, temporal, and spectral resolution.

In this Letter, we explore ion equilibration during the first microsecond after the plasma is created. The density sets the time and the energy scale for equilibration, but electron screening effects are evident. Even when the number of electrons per Debye sphere is small, the equilibration temperature of the ions agrees with a model [15] that uses a Yukawa ion-ion potential.

We also observed oscillations of the ion kinetic energy. For many years, this phenomenon has been the subject of intense study through analytic calculations [7] and simulations [6,8-12] of one-component strongly coupled plasmas, but it has not previously been observed experimentally. The oscillations and their damping reflect universal dynamics of a Coulomb system with spatial correlations.
PACS numbers: $52.27 . \mathrm{Gr}, 32.80 . \mathrm{Pj}, 52.38 . \mathrm{Dx}, 52.70 . \mathrm{Kz}$

Details on laser cooling, plasma formation, and imaging are given in $[14,16]$. The experiment starts with strontium atoms that are cooled and trapped in a magneto-optical trap (MOT). The neutral atom cloud is characterized by a temperature of about $10 \mathrm{mK}, 2 \times 10^{8}$ atoms, and a Gaussian density distribution. We vary the atom density by changing the MOT parameters, or by turning the MOT off and releasing the atoms in a ballistic expansion. Up to $30 \%$ of the neutral atoms are then ionized with one photon from the cooling laser and one photon from a pulsed dye laser. The ion density distribution equals the atom distribution at the time of photoionization and is given by $n_{i}(r)=n_{0 i} \exp \left(-r^{2} / 2 \sigma^{2}\right)$, with $\sigma$ from 0.6 to $1 \mathrm{~mm}$ and $n_{0 i}$ from $2 \times 10^{9}$ to $1.4 \times 10^{10} \mathrm{~cm}^{-3}$. The electron density, $n_{e}(\mathbf{r})$, closely follows the ion density, and the initial electron temperature is given by $T_{e}=2 E_{e} / 3 k_{B}$, where $E_{e}$ is the detuning of the pulsed laser above the ionization threshold.

At an adjustable delay time $\left(t_{\text {delay }}\right)$ after photoionization, a collimated laser beam, tuned near resonance with the $\mathrm{Sr}^{+}$ ${ }^{2} S_{1 / 2}-{ }^{2} P_{1 / 2}$ transition at $422 \mathrm{~nm}$, illuminates the plasma and falls on an intensified charge coupled device camera. By varying $t_{\text {delay }}$, we study the evolution of the plasma after formation.

The optical depth (OD) for a laser propagating along the $z$ axis is defined by $\operatorname{OD}(x, y)=\log \left[I_{\text {background }}(x, y) /\right.$ $\left.I_{\text {image }}(x, y)\right]$, where $I_{\text {background }}$ and $I_{\text {image }}$ are laser intensities without and with the plasma present. By integrating the optical depth over $x$ and $y$ for images taken at different image beam frequencies, $\nu$, we obtain the absorption spectrum

$$
\begin{aligned}
S(\nu) & =\int d x d y \mathrm{OD}(x, y)=\int d^{3} r n_{i}(r) \alpha\left[\nu, T_{i}(r)\right] \\
& \equiv N_{i} \alpha\left(\nu, T_{i, \mathrm{eff}}\right) .
\end{aligned}
$$

We have used Beer's law to relate $S(\nu)$ to the absorption cross section, $\alpha\left[\nu, T_{i}(r)\right]$, and the ion density. The absorption cross section is a function of temperature due to the Doppler broadening, and since we expect the temperature to vary with density, we allow $\alpha$ to vary with position. The 
absorption cross section has a Voigt profile resulting from the convolution of the Gaussian Doppler broadening with a Lorentzian with width $\gamma=\gamma_{0}+\gamma_{\text {laser }}$. Here, $\gamma_{0}=$ $22 \mathrm{MHz}$ is the natural width of the transition, and $\gamma_{\text {laser }}=$ $(5 \pm 2) \mathrm{MHz}$ is the measured linewidth of the laser. As shown in the second line of Eq. (1), we fit the spectrum to a single Voigt profile with effective temperature $T_{i, \text { eff }} . N_{i}$ is the number of ions. We will discuss below how $T_{i, \text { eff }}$ relates to the average ion temperature in the plasma.

Figure 1(a) shows the evolution of $T_{i \text {,eff }}$ for three different densities. The rapid increase in the temperature for $t_{\text {delay }}<300 \mathrm{~ns}$ is due to disorder-induced heating. This originates from conversion of Coluomb potential energy into kinetic energy as the ions evolve from a completely disordered state to one with some degree of spatial correlations. This was predicted in [15] and observed in numerical simulations [12,17,18] and the first experimental studies with optical imaging [14].

The time scale of the heating is the inverse ion plasma frequency $\omega_{p i}^{-1}=\sqrt{m_{i} \varepsilon_{0} / n_{i} e^{2}} \approx 100 \mathrm{~ns}$. The energy scale is $T_{C}=e^{2} / 4 \pi \varepsilon_{0} a k_{B} \approx 5 \mathrm{~K}$, where $a=\left(4 \pi n_{i} / 3\right)^{-1 / 3}$ is the Wigner-Seitz radius. Figure 1(b) shows data with time scaled by the average inverse plasma frequency and temperature scaled by $T_{C}$. The three curves coincide very well in the time axis, but show slight deviation in the temperature axis. The deviation indicates the effects of electron screening of the ion-ion interaction.

A detailed expression for the equilibrium ion temperature, including screening effects, was derived in [15]. Assuming complete disorder and stationary ions at $t_{\text {delay }}=$ 0 , and incorporating electron screening through a Yukawa ion-ion potential $[19,20]$,

$$
T_{i}=\frac{2}{3} \frac{e^{2}}{4 \pi \varepsilon_{0} a k_{B}}|\tilde{U}+\kappa / 2| .
$$

Here, $\kappa=a / \lambda_{D}$, where $\lambda_{D}=\left(\varepsilon_{0} k_{B} T_{e} / n_{i} e^{2}\right)^{1 / 2}$ is the Debye length. The quantity $\tilde{U} \equiv U /\left(N_{i} e^{2} / 4 \pi \varepsilon_{0} a\right)$ is the potential energy per particle in units of $e^{2} / 4 \pi \varepsilon_{0} a$, and it has been tabulated in [19]. Equation (2) is complicated by the fact that $\tilde{U}$ is a function of $n_{i}$ and $T_{i}$. From the table of
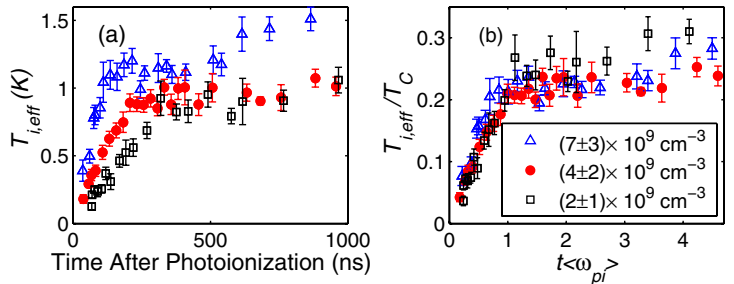

FIG. 1 (color online). The effective ion temperature, $T_{i \text {,eff }}$, versus time after photoionization for an initial electron temperature of $T_{e}=2 E_{e} / 3 k_{B}=38 \pm 6 \mathrm{~K}$ and various plasma densities. (a) The data are plotted on absolute temperature and time scales. (b) The time is scaled by the inverse of the average plasma period, and $T_{i, \text { eff }}$ is scaled by $T_{C}$.
$\tilde{U}$ and using an iterative numerical routine, $T_{i}$ can be obtained for a given $n_{i}$ and $T_{e}$.

The effects of electron screening are quantified by the factor $|\tilde{U}+\kappa / 2|$. Colder electron temperature and higher density lead to a smaller Debye length and greater screening of the ion-ion potential. This decreases the potential energy in the initial system, and thus, compared to a system in which there is no screening, decreases the equilibration temperature reached. This effect is evident in Fig. 2, which shows data from three different initial electron energies but the same ion density distribution. To quantitatively compare the data in Figs. 1 and 2 with Eq. (2) we must account for two factors. The first is the effect of the inhomogeneous density distribution. We expect the ion temperature to vary with density because global thermal equilibrium occurs on a hydrodynamic time scale, $\sigma / v$, which is on the order of tens of $\mu \mathrm{s}$, where $v$ is the ion acoustic wave velocity. Local thermal equilibration occurs on a much faster time scale, $\sim \omega_{p i}^{-1}$ [21]. If we assume a temperature distribution given by Eq. (2), numerical simulations [22] show that $T_{i \text {,eff }}=(0.95 \pm$ 0.05) $T_{i, \mathrm{av}}$. Here $T_{i, \mathrm{av}}$ is the average ion temperature found by averaging Eq. (2) over the density profile.

To extract the average ion temperature, we must also account for acceleration of the ions by the electron pressure. This was studied experimentally in $[14,23]$ and theoretically in [24,25], and it is evident in the continuing increase in $T_{i, \text { eff }}$ in Figs. 1 and 2 for $t_{\text {delay }}>500$ ns. This acceleration will lead to expansion of the plasma after a time on the order of $10 \mu \mathrm{s}$, and although it is not a heating effect, it contributes to the Doppler width of the spectrum. Numerical analysis [22], assuming a temperature distribution given by Eq. (2), shows that the effect of plasma expansion on the effective ion temperature can be approximated by

$$
T_{i, \text { eff }}\left(t_{\text {delay }}\right)=C T_{i, \text { av }}\left[1+1 / C\left(t_{\text {delay }} / t_{\text {exp }}\right)^{2}\right]
$$

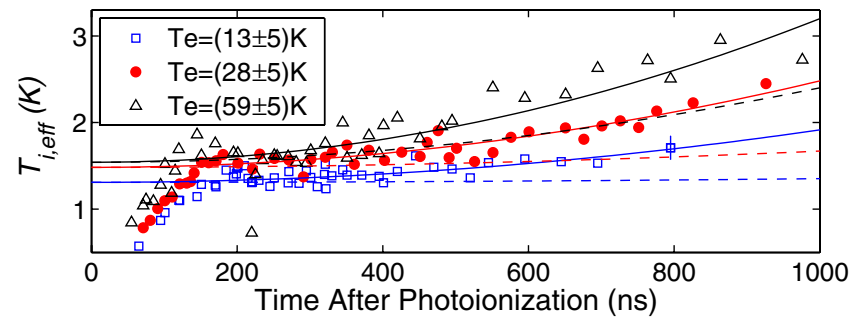

FIG. 2 (color online). Effective ion temperature versus time after photoionization for various electron temperatures. The peak ion density for all data is $n_{0 i}=(1.4 \pm 0.5) \times 10^{10} \mathrm{~cm}^{-3}$. The solid curves are fits of the data based on Eq. (3). The fit determines the equilibrium $T_{i, \text { av }}$ and $T_{e}$. The fit $T_{e},(82 \pm 5)$, $(64 \pm 6)$, and $(45 \pm 6) \mathrm{K}$ are higher than the expected initial $T_{e}$ shown in the legend, indicating electron heating. The dashed curves show the increase in $T_{i \text {,eff }}$ due to radial acceleration expected for $T_{e}=2 E_{e} / 3 k_{B}$. A typical data error bar is shown. 
where $C=(0.95 \pm 0.05)$ is a constant and $t_{\exp }$ is the time scale when the Doppler broadening contributed by plasma expansion is comparable to the thermal Doppler broadening. It is given by

$$
t_{\exp }=\sigma \sqrt{m_{i} / k_{B} T_{e}} \sqrt{T_{i, \text { av }} / T_{e}} .
$$

Because we want to check the accuracy of Eq. (2), and we assume this equation in our analysis, strictly speaking, our strongest conclusion can be that our data are consistent with Eq. (2). However, we found [22] that Eq. (3) is accurate for a range of temperature distributions, from global thermal equilibrium to local thermal equilibrium at a temperature expected in the absence of electron screening. It can be shown analytically that Eq. (3) has the correct long-time limit for when the plasma reaches global equilibrium, or when the expansion velocity greatly exceeds the thermal velocity. We do not believe our analysis is very sensitive to a particular assumption of $T_{i}(r)$.

In Fig. 2, using Eq. (3), we extract $T_{i, \text { av }}$ and $T_{e}$ from the data. The model addresses the dynamics after local equilibration, not the disorder-induced heating phase during the first $100 \mathrm{~ns}$. The fit $T_{e}$ exceed $2 E_{e} / 3 k_{b}$ for the data shown. As predicted by theory $[17,18,24,26]$, this effect becomes larger as the initial Coulomb coupling parameter for the electrons, $\quad \Gamma_{e}=\kappa^{3} / 3=e^{2} /\left(4 \pi \varepsilon_{0} a k_{B} T_{e}\right), \quad$ approaches unity. $\left(\Gamma_{e}=0.5\right.$ for the coldest electron temperature data in Fig. 2, assuming $T_{e}=2 E_{e} / 3 k_{B}$ and using the peak density.) Likely mechanisms for heating are recombination, continuum lowering, and disorder-induced electron heating. We plan to study this phenomenon in the future, but our main goal now is accurate determination of $T_{i, \mathrm{av}}$.

The inferred value of $T_{i, \text { av }}$, which is the average ion temperature after the plasma reaches local thermal equilibrium, is shown in Fig. 3. The uncertainty in $T_{i, \text { av }}$, of about $0.2 \mathrm{~K}$, is determined by the statistical noise in the data and the uncertainty in $C$. We also show the theoretical value of $T_{i, \text { av }}$, found by averaging Eq. (2) over the density distribution. Uncertainty in this quantity results from uncertainty in the value of the peak density. Experimental and
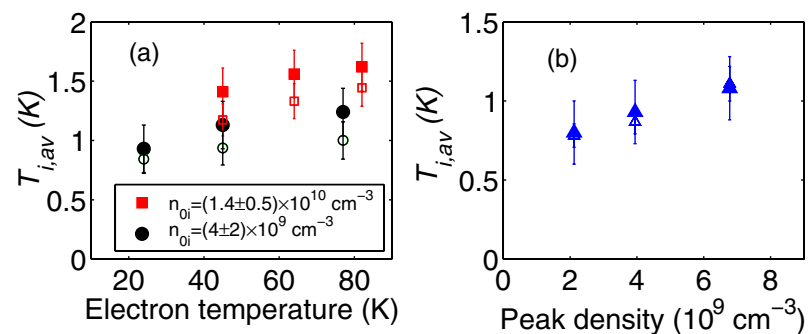

FIG. 3 (color online). The comparison between the data and the theoretical calculations. In (a), the density is fixed and the initial $T_{e}$ is varied. Solid symbols are experimental data and open symbols are theoretical calculations. In (b), the initial $T_{e}$ is fixed $(38 \pm 6 \mathrm{~K})$, while the density is varied. The $T_{e}$ for all theoretical calculations are based on the fit $T_{e}$. theoretical results agree well. It is not initially surprising that Eq. (2) accurately predicts the ion temperature for equilibrating ultracold neutral plasmas. The theory basically expresses energy conservation, as emphasized in [12]. However, for the data in Fig. 3 with the lowest fit $T_{e}$ and highest $n_{0 i}$, the peak value of $\kappa$ in the plasma is 0.7 . This corresponds to three electrons per Debye sphere $\left(\kappa^{-3}=n_{e} 4 \pi \lambda_{D}^{3} / 3\right)$. One might not necessarily expect Eq. (2) to be accurate in this regime because it assumes a Yukawa potential for ion-ion interactions. The Yukawa potential is based on Debye screening and is normally derived for $\kappa^{-3} \gg 1$.

From the measured $T_{i, \mathrm{av}}, T_{e}$, and density distribution, we calculate the average Coulomb coupling constant for Debye-screend ions, $\Gamma_{i, \text { av }}^{*}=\left\langle\exp [-\kappa(r)] e^{2} /\right.$ $\left.\left[4 \pi \varepsilon_{0} a(r) k_{B} T_{i, \text { av }}\right]\right\rangle$. For all the data shown in Fig. $3, \Gamma_{i, \text { av }}^{*}$ is in the range of 1.7 to 2.5 . With lower $T_{e}$ and higher density, $\Gamma_{i, \text { av }}^{*}$ is slightly higher. The surprisingly small variation in $\Gamma_{i \text {,av }}^{*}$ suggests that disorder-induced heating is a natural feedback mechanism that leads to equilibration just barely in the strongly coupled regime.

Close inspection of Figs. 1 and 2 reveals that at the end of the disorder-induced heating phase the ion temperature overshoots its equilibrium value before settling. This phenomenon is more evident in Fig. 4(a), where $T_{i, \text { av }}$ is calculated for an inner and outer region of the plasma image $\left(\rho=\sqrt{x^{2}+y^{2}}<0.9 \sigma\right.$ and $\rho>1.48 \sigma$, respectively). Each selected annular region contains $1 / 3$ of the ions and probes a region with significantly less variation in density than in the entire plasma. The region with lower density has lower $T_{i, \text { av }}$, supporting our hypothesis of local thermal equilibrium, but the oscillation is the most striking observation.

Intuitively, one can explain this phenomenon as an oscillation of each ion in its local potential energy well. A simple calculation implies that the time for an ion to move an interparticle distance, when accelerated from rest by a force of $e^{2} /\left(4 \pi \varepsilon_{0} a^{2}\right)$, varies as $\sim \omega_{p i}^{-1}$, and the observed oscillation occurs at $2 \omega_{p i}$. As expected from the density dependence of $\omega_{p i}$, the oscillation period is longer
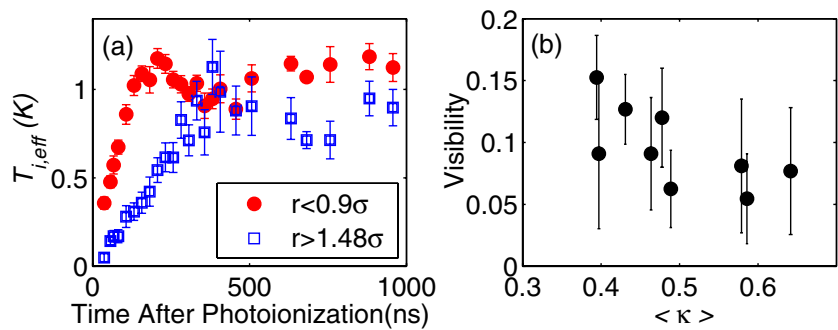

FIG. 4 (color online). Effective ion temperature obtained from different selected regions of the cloud for $n_{0 i}=(4 \pm 2) \times$ $10^{9} \mathrm{~cm}^{-3}$ and initial $T_{e}=2 E_{e} / 3 k_{B}=38 \pm 6 \mathrm{~K}$. In (b), we plot the visibility of the oscillation for the central probed region versus the averaged $\kappa$ for this region for all our data taken at different conditions. 
for the outer region where the average density is lower. This explains why averaging over the entire cloud obscures the oscillation; the motion dephases because of the variation in $\omega_{p i}$. It is questionable whether the ion motion should be called an ion plasma oscillation because there is probably no collective or long range coherence to the motion.

Another way to think of the oscillation is relaxation of the two-particle distribution function in the plasma, which describes two-particle spatial and momentum correlations. Disorder-induced heating and oscillation of the kinetic and the potential energy represent equilibration of spatial correlations. Normally, the time scale for equilibration of the two-particle distribution function is much shorter than the time scale for the one-particle distribution function to reach local thermal equilibrium. This is known as Bogoliubov's hypothesis [27]. For strongly coupled plasmas, however, these time scales both become equal to $\omega_{p i}^{-1}[9]$.

Kinetic-energy oscillations at $2 \omega_{p i}$ have been observed in molecular dynamics simulations of equilibrating strongly coupled systems [9,11,12]. Calculations [6,28] also show oscillations in the velocity autocorrelation function in equilibrium strongly coupled one-component plasmas. To our knowledge, this is the first experimental observation of the phenomenon.

Numerical results [9] suggest that the damping time for the oscillations is approximately $\pi / \omega_{p i}$ for $\Gamma_{i} \geq 5$, and that lower $\Gamma_{i}$ leads to faster damping. Because our analysis still averages over the $z$ axis of the plasma, which introduces dephasing into the observed oscillations, it is difficult to comment on the damping of the oscillation. But we do find a correlation between the visibility of the oscillation and $\kappa$, as shown in Fig. 4(b), suggesting that electron screening plays a role in the damping. The visibility is defined as $\left(T_{i \text {,eff }}^{\text {peak }}-T_{i \text {,eff }}^{\text {dip }}\right) /\left(T_{i, \text { eff }}^{\text {peak }}+T_{i, \text { eff }}^{\text {dip }}\right)$, where $T_{i, \text { eff }}^{\text {peak }}$ and $T_{i \text {,eff }}^{\mathrm{dip}}$ are effective ion temperatures at the peak and the dip of the oscillation.

In conclusion, we have studied the equilibration of ultracold neutral plasmas. The screening effect of electrons on the final ion temperature is observed, and experiment and theory agree very well. As predicted from numerical simulations, the ion kinetic energy displays oscillations that are characteristic of equilibrating strongly coupled plasmas.

This research was supported by the Department of Energy, Office of Fusion Energy Sciences, National Science Foundation, Office for Naval Research, Research Corporation, Alfred P. Sloan Foundation, and David and Lucille Packard Foundation.
[1] H. M. V. Horn, Science 252, 384 (1991).

[2] T. B. Mitchell, J. J. Bollinger, X. P. Huang, W. M. Itano, and D. H. E. Dubin, Phys. Plasmas 6, 1751 (1999).

[3] J. H. Chu and L. I, Phys. Rev. Lett. 72, 4009 (1994).

[4] M. Nantel, G. Ma, S. Gu, C. Y. Cote, J. Itatani, and D. Umstadter, Phys. Rev. Lett. 80, 4442 (1998).

[5] E. Springate, N. Hay, J. W. G. Tisch, M. B. Mason, T. Ditmire, M.H.R. Hutchinson, and J.P. Marangos, Phys. Rev. A 61, 063201 (2000).

[6] J. P. Hansen, E. L. Pollock, and I. R. McDonald, Phys. Rev. Lett. 32, 277 (1974).

[7] H. Gould and G. F. Mazenko, Phys. Rev. Lett. 35, 1455 (1975).

[8] M. Bonitz, D. Semkat, and D. Kremp, Phys. Rev. E 56, 1246 (1997).

[9] G. Zwicknagel, Contrib. Plasma Phys. 39, 155 (1999).

[10] K. Morawetz, M. Bonitz, V. G. Morozov, G. Röpke, and D. Kremp, Phys. Rev. E 63, 020102 (2001).

[11] I. V. Morozov and G.E. Norman, J. Phys. A 36, 6005 (2003).

[12] T. Pohl, T. Pattard, and J.M. Rost, J. Phys. B 37, 183 (2004).

[13] T. C. Killian, S. Kulin, S. D. Bergeson, L. A. Orozco, C. Orzel, and S. L. Rolston, Phys. Rev. Lett. 83, 4776 (1999).

[14] C.E. Simien, Y.C. Chen, P. Gupta, S. Laha, Y. N. Martinez, P. G. Mickelson, S. B. Nagel, and T. C. Killian, Phys. Rev. Lett. 92, 143001 (2004).

[15] M. S. Murillo, Phys. Rev. Lett. 87, 115003 (2001).

[16] S. B. Nagel, C. E. Simien, S. Laha, P. Gupta, V. S. Ashoka, and T. C. Killian, Phys. Rev. A 67, 011401 (2003).

[17] S. G. Kuzmin and T. M. O’Neil, Phys. Plasmas 9, 3743 (2002).

[18] A. N. Tkachev and S.I. Yakovlenko, Quantum Electron. 30, 1077 (2000).

[19] S. Hamaguchi, R. T. Farouki, and D. H. E. Dubin, Phys. Rev. E 56, 4671 (1997).

[20] D. H. E. Dubin and T. M. O’Neil, Rev. Mod. Phys. 71, 87 (1999).

[21] T. Pohl, T. Pattard, and J. M. Rost, Phys. Rev. A 70, 033416 (2004).

[22] T. C. Killian, Y. C. Chen, P. Gupta, S. Laha, Y. N. Martinez, P. G. Mickelson, S. B. Nagel, A. D. Saenz, and C. E. Simien, physics/0407138.

[23] S. Kulin, T. C. Killian, S. D. Bergeson, and S. L. Rolston, Phys. Rev. Lett. 85, 318 (2000).

[24] F. Robicheaux and J. D. Hanson, Phys. Plasmas 10, 2217 (2003).

[25] T. Pohl, T. Pattard, and J. M. Rost, Phys. Rev. Lett. 92, 155003 (2004).

[26] S. Mazevet, L. A. Collins, and J. D. Kress, Phys. Rev. Lett. 88, 55001 (2002).

[27] D. R. Nicholson, Introduction to Plasma Theory (Krieger Publishing Company, Malabar, FL, 1992).

[28] J.P. Hansen, I. R. McDonald, and E. L. Pollock, Phys. Rev. A 11, 1025 (1975). 